\title{
Theoretical derivation of critical current density and critical magnetic field considering many-body interactions of magnetic flux quanta
}

\author{
Shinichi Ishiguri, Ph.D. \\ Nihon University \\ 1-2-1 Izumi-Cho, Narashinoshi, Chiba 275-8575 JAPAN \\ TEL: +81-47-474-9143 \\ Email: shinichi.ishiguri@gmail.com
}

\begin{abstract}
To clarify the relationships among critical temperature, critical magnetic field, and critical current density, this paper describes many-body interactions of quantum magnetic fluxes (i.e., vortices) and calculates pinning-related critical current density. All calculations are analytically derived, without numerical or fitting methods. After calculating a magnetic flux quantum mass, we theoretically obtain the critical temperature in a many-body interaction scenario (which can be handled by our established method). We also derive the critical magnetic field and inherent critical current density at each critical temperature. Finally, we determine the pinning-related critical current density with selffields. The relationships between the critical magnetic field and critical temperature, inherent critical current density and critical temperature, and pinning critical current density and self-magnetic field were consistent with experimental observations. From the critical current density and critical magnetic field, we clarified the magnetic field transition. It appears that a magnetic flux quantum collapses when the lattice of magnetic flux quanta melts. Our results, combined with our previously published papers, provide a comprehensive understanding of the transition points in high- $T_{\mathrm{c}}$ cuprates.
\end{abstract}

\section{Keywords}

vortices, magnetic flux quantum, critical current density, critical magnetic field, many-body interaction

\section{Introduction}

First, we briefly review the history of superconductors. Mercury was recognized as the first superconductor over 100 years ago, sparking worldwide interest among theoreticians and experimentalists. The Bardeen-Cooper-Schrieffer theory describes low-temperature superconducting metals [1]. After that, high-Tc cuprates [2], $\mathrm{MgB}_{2}$ [3], and Fe-based superconductors [4] were found. 
Theoretical explanations of high-Tc cuprates have been attempted with little success, although other superconductors have provided theoretical insights. High-Tc cuprates were analytically described in our previous paper [5]. Very recently, H-based superconductors with extremely high pressures [6] have been reported.

Interest in the superconductors mentioned above was triggered by the high critical temperatures of these materials. However, a typical superconductor has three critical points: a critical temperature, a critical magnetic field, and a critical current density. The critical magnetic field and critical current density are directly related to magnetic flux quanta dynamics [7]. When a superconductor is exposed to a magnetic field, the flux is quantized as $h / 2 e$, where $h$ and $e$ are the Planck constant and electron charge, respectively [8]. Therefore, the magnetic field distribution is non-uniform below the transitions. Furthermore, the three critical points are interdependent, and their relationships can be explained by theoretical approaches [9]. However, magnetic flux quanta, which undertake many-body interactions that are difficult to describe analytically, underpin these relationships. Thus, the transition points are usually derived through numerical calculations or fitting methods.

Besides the pure critical current, there is a critical current associated with pinning forces. The pinning center implies impurities are distributed among a superconductor's substance. Experimentally, some superconductors exhibit higher critical current density when artificially doped with impurities. This behavior occurs when a magnetic flux quantum is pinned at a pinning center. Although the pinning critical current density is related to the pinning center, we claim that the prevailing dogma contains some inaccuracies. Specifically, the critical current density undergoes a transition at some proportion of the pinning force to the Lorentz force, when a magnetic flux quanta is received by a current. However, this picture is contentious because it does not describe the collapse of the magnetic flux quantum, which is essential for explaining the observed uniformity of the magnetic field distribution after the transition. Moreover, if a magnetic flux quantum receives the Lorentz force from another point in the sea of current, then the sea of current must experience a reaction force. However, the point in the sea which receives the reaction forces cannot be determined. Finally, the critical current density does not admit a theoretical solution under the conventional pinning assumption and can be solved only by fitting or numerical methods. These methods measure the critical current and only estimate the pinning force, which is insufficient.

In high-Tc cuprates, the critical current density depends on the applied magnetic field, the magnetic field angle, and temperature. Approximately ten years ago, we showed theoretically this critical current depends on the applied magnetic fields and their angles at $77 \mathrm{~K}$ [10]. To demonstrate a critical current with a self-field, however, we must handle many-body interactions of the magnetic flux quanta; we did not attempt it in our previous work. Recently, reagarding high-Tc cuprates, we obtained the critical 
temperature, the pseudo-gap temperature, and the transition temperatures at which anomalous metal phases appear on doping [5]. We found a theoretical, general, and novel statistical method that handles many-body interactions in this work. This method obtains the above three transition temperatures without numerical or fitting methods. Accepting this finding and the result of our ten-year-prior work [10], the present paper describes the critical current and critical magnetic field at critical temperature and determines their interdependent relationships. Furthermore, the pinning critical current density of a self-magnetic field is derived. Similar to our previous work on high-Tc cuprates, the critical current density is calculated using physical constants rather than numerical or fitting techniques. As shown in the Results section, the derived critical current densities agree well with measurements.

The significances of the present paper are summarized below. Details are given in the Discussion section.

1) Critical currents are theorized without fitting methods or numerical calculations.

2) The physical picture of the superconducting transition under magnetic fields is clarified.

3) The relationships among critical current, critical magnetic field, and critical temperature are theoretically revealed.

The remaining contents of the present paper are organized as follows.

First, we review our critical current derivation under an applied magnetic field derived in our ten-yearold paper. We then describe the statistical method that handles many-body interactions. This method appeared in our previous paper on the transition temperatures in high-Tc cuprates.

Section 2 is followed by the theory section, in which we describe the mass of a magnetic flux quantum, the phase transition, and the derivation of critical current density. Sections 4 and 5 present the results and discussion, respectively. The paper concludes with Section 6.

\section{Review section}

\subsection{Derivation of critical current density under an applied magnetic field}

\subsubsection{Introduction}

The critical current densityof a high-temperature superconductor (HTS) tape wire depends on the applied magnetic field and magnetic field angle at a constant temperature. These dependencies were formulated in our previous paper on HTS tape wire [10]. In our previous paper, a wavefunction at magnetic field transitions was derived. The present paper reuses the same 
wavenumber of the wavefunction to formulate the equations. Also, reusing our earlier results, we include the pinning critical current with a self -magnetic field. Let us review this previous paper before proceeding.

\subsubsection{Outline}

We assume a relatively large magnetic field and thus a sufficiently large number of pinning centers or lattices of magnetic flux quanta.

We first discuss the local flux quantum, whose value must remain as $h / 2 e$, and is surrounded by a shield current. When the applied magnetic field increases, the radius of the flux quantum must decrease to maintain the constant value $h / 2 e$. We assume that this radius corresponds to that of the superconducting shield current surrounding the flux quantum. In short, increasing the applied magnetic field reduces the radius of the superconducting loop-shielding current. Such variation of the loop-current radius is evidenced by the increased density of magnetic flux quanta over a sample substance when the radius of the superconducting loop current decreases.

As the applied magnetic field increases, the radius of the surrounding current eventually corresponds to that of a pinning potential. The superconducting loop current disappears at magnetic fields beyond this transition point because the pinning potential prevents further decrease of the radius. The shape of the flux quantum then collapses, and the tuniform magnetic field expands throughout the material, as described later.

\subsubsection{Wave function at the transition}

The loop current surrounding a magnetic flux quantum indirectly implies the rotational movement of a Cooper pair. Therefore, when discussing the loop current along with a flux quantum, we consider the wave function around the flux quantum, which is given by [11]

$$
\Phi=|\Phi| \exp (j \varphi),
$$

where

$$
\begin{aligned}
& \varphi=\oint \frac{q \vec{A}}{\hbar} \cdot d \vec{r}, \\
& \varphi=2 n \pi .
\end{aligned}
$$

These equations are determined in cylindrical coordinates, and $q, j, A$, and $n$ denote the charge of a pair, the imaginary unit, the vector potential, and an integer, respectively. Therefore, the quantized flux is given by

$$
\int \vec{B} \cdot d \vec{S}=\int \operatorname{rot} \vec{A} \cdot d \vec{S}=\oint \vec{A} \cdot d \vec{r}=\frac{h}{2 e} \text { for } n=1 .
$$


The center of a pin corresponds to the origin of the cylindrical coordinates. The pinning potential is approximated as a cylindrical square-well potential. From Eq. (2), the wavenumber along the circle is

$$
k=\frac{q A}{\hbar} .
$$

Once the radius of the loop current coincides with that of the pinning potential, further increases of the magnetic field cause the radius of the loop current to decrease, and the pinning potential induces a collision of the Cooper pair producing this current. The pair then breaks.

As Eqs. (1) and (2) indicate the center-of-mass motion of a Cooper pair. The wavenumber reduces by half when the Cooper pair is destroyed:

$$
k_{n}=\frac{q A}{2 \hbar} .
$$

After the destruction of a Cooper pair (i.e., at the transition point and beyond), the wave function cannot be a plane wave. The wave function at the transition is derived as follows. The electrons move in the direction of the vector potential $A$ (i.e., tangential to the current circle), as shown in Fig. 1.

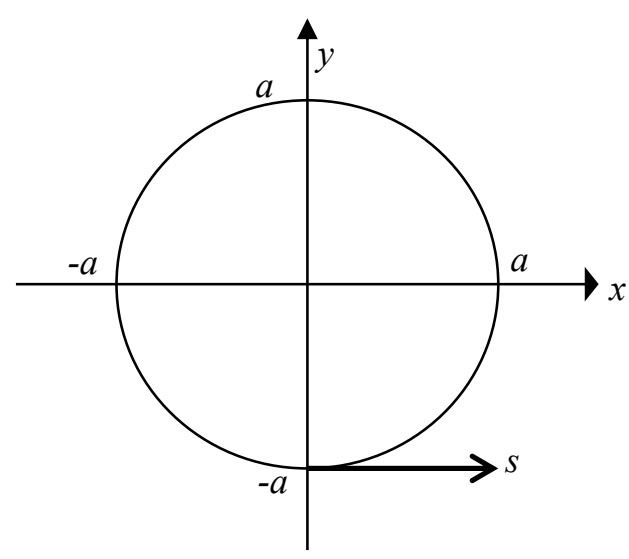

Fig. 1

Schematic of distribution of unpaired electrons around a pinning potential ( $a$ denotes the radius of the magnetic flux quantum)

The movements of all electrons along the circle must cease simultaneously. However, because an electron can still be considered as a wave, its wavelength must be conserved; consequently, the wave function at the transition decays to $1 / e$ of its original value:

$$
\psi=|\Phi| \exp \left(-\frac{1}{\lambda}|x|\right)
$$


where $\lambda$ denotes the electron wavelength, determined by inverting the wavenumber. The wave function is

$$
\psi=|\Phi| \exp \left(-\frac{e A}{2 \hbar}|x|\right)
$$

where $e$ is the electron charge.

We consider high-Tc cuprates, whose $\mathrm{CuO}_{2}$ surfaces form a two-dimensional (2D) current sheet $[11,12]$.

If the applied magnetic field is perpendicular to the $2 \mathrm{D}$ current sheet of the substance, the wave function is:

$$
\psi_{1}=|\Phi| \exp \left(-\frac{e B_{s} a}{2 \hbar}|x|\right)
$$

where $a$ is the radius of the magnetic flux quantum and $B_{s}$ is a magnetic field component perpendicular to the 2D sheet. This study assumes a cylindrical pin. As described earlier, the de-paired electrons are distributed across the pin potential, so their positions reach the radius $a$. Eq. (9) then becomes

$$
\psi_{1}=|\Phi| \exp \left(-\frac{e a^{2} B_{S}}{2 \hbar}\right) .
$$

When the electron concentration is proportional to $\left|\psi_{1}\right|^{2}$, the current density is

$$
j_{c 1}=p e v \times \exp \left(-\frac{e a^{2}}{\hbar} B_{S}\right),
$$

where $p$ and $v$ are the proportionality constant and velocity, respectively.

A similar calculation is performed when the electrons move parallel to the $2 \mathrm{D}$ current sheet. We thus obtain

$$
j_{c 2}=p e v \times \exp \left(-\frac{e a d}{\hbar} B_{t}\right),
$$

where $B_{t}$ is the magnetic field component parallel to the $2 \mathrm{D}$ current sheet and $d$ is the width of the current sheet.

Combining the two current densities [10], the critical current density is

$$
j_{c}=j_{c 0} \exp \left[-\left(\frac{e a^{2}}{\hbar} B_{s}+\frac{e a d}{\hbar} B_{t}\right)\right],
$$

where $j_{\mathrm{c} 0}$ denotes a constant current density.

In terms of the magnitude $B$ and angle $\theta$ of the magnetic field, the critical current density becomes

$$
j_{c}=j_{c 0} \exp \left[-B\left(\frac{e a^{2}}{\hbar} \sin \theta+\frac{e a d}{\hbar} \cos \theta\right)\right]
$$

\subsubsection{Results of this review section}

To confirm the derived critical current equation's accuracy, we compared its results with the measured current density along a YBCO tape wire at $77 \mathrm{~K}$. The comparison results at magnetic fields of $0.5,1.0$, 
and 2.0 T are shown in Figs. 2, 3, and 4, respectively. The theoretical and measured values were in good agreement. The measurement method and data of the YBCO tape are given in [13].

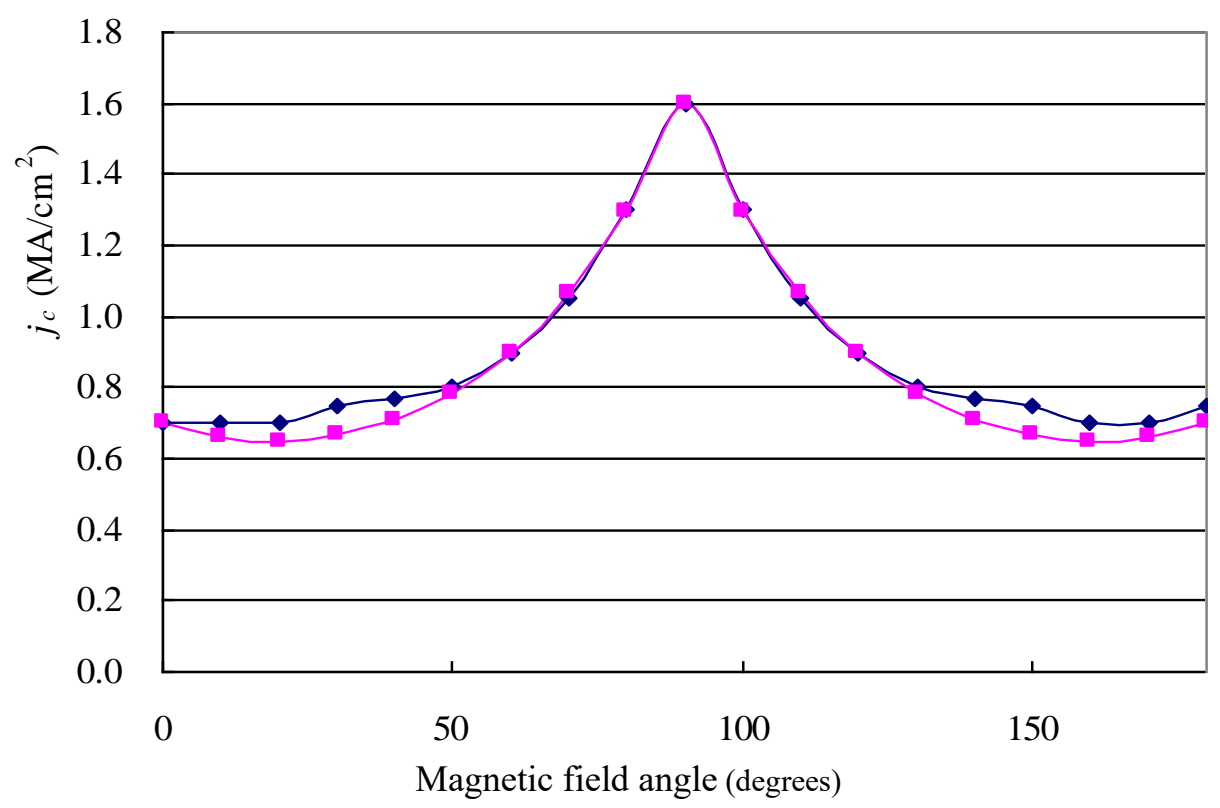

Fig. 2

Theoretical (pink) and measured (dark blue) current densities in YBCO tape wire versus magnetic field angle at $B=$ $0.5 \mathrm{~T}$.

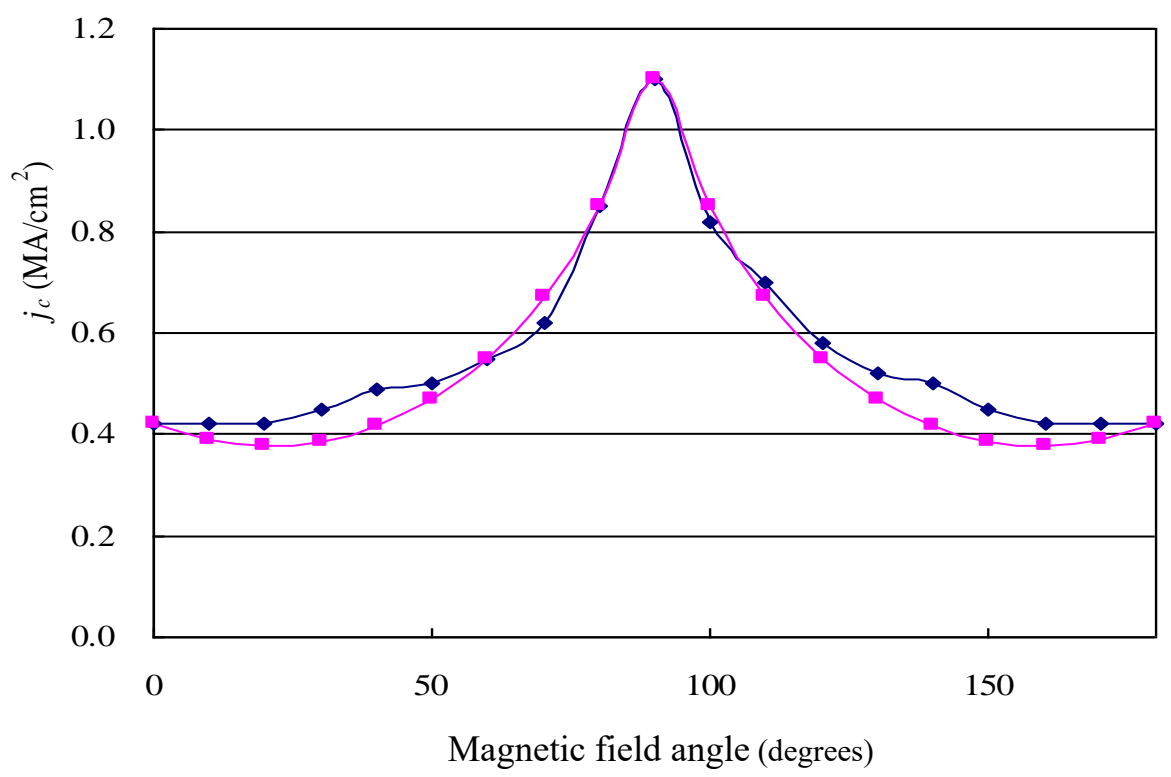


Fig. 3

Theoretical (pink) and measured (dark blue) current densities in YBCO tape wire versus magnetic field angle at $B=$ $1.0 \mathrm{~T}$

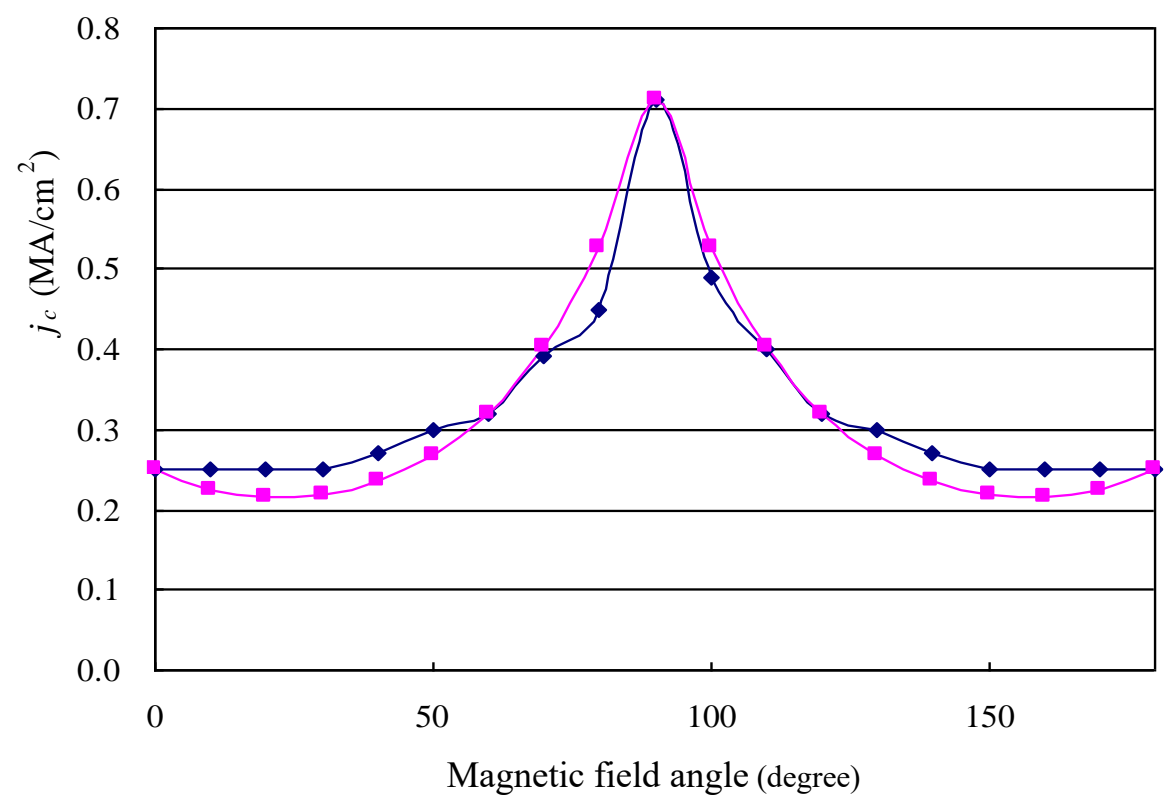

Fig. 4

Theoretical (pink) and measured (dark blue) current densities in YBCO tape wire versus magnetic field angle at $B=$ $2.0 \mathrm{~T}$

\subsection{Review of the statistical method for many-body interactions}

To consider a transition with many-body interactions, we must recall our original model [5]. First, let us review this model, including Coulomb interactions. A many-body interaction implies that each location $i$ has a local temperature $T_{i}$. We first consider a spherical shell containing a charged particle such as an electron (see Fig. 5). In this model, the local thermal equilibrium temperature $T_{i}$ exists only within a differential number $\mathrm{d} N$. Note that $N$ is sufffiently large number and thus $\mathrm{dN}$ implies its differential. 


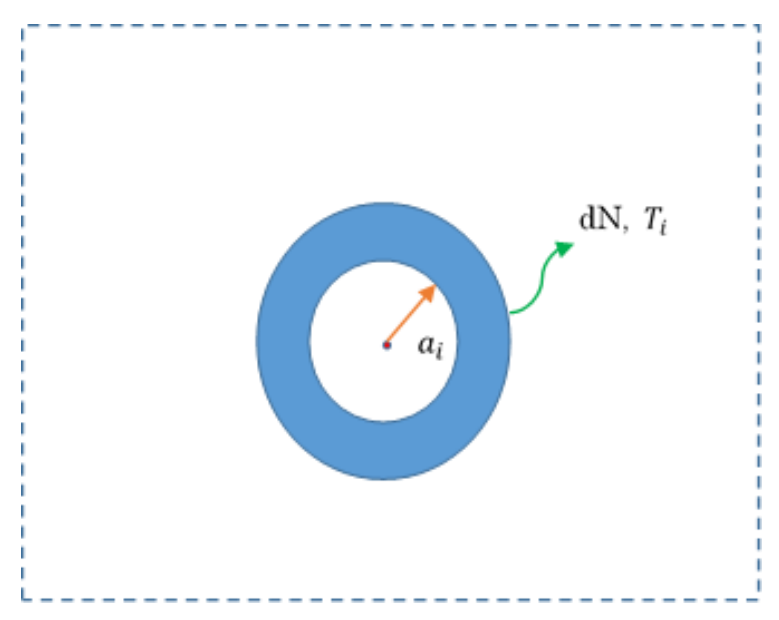

Fig. 5

Schematic of our established model to handle many-body interactions to produce a statistic equation. With consideration of the nature of many-body interactions, note that temperatures are locally different. However, this model implies that, only in differential number $\mathrm{dN}$ (a carrier takes the center and $\mathrm{dN}$ takes a temperature $T_{i}$ ), a thermal equilibrium can be presumed. Therefore, when considering two forces that are experienced by $\mathrm{dN}$, a proportion between the force of expansion from Coulomb interactions and the force of compression from immediate outer side, which is equal to the kinetic energies in $\mathrm{dN}$ (i.e., a temperature $T_{i}$ ), is formed. By calculating this proportional equation, a new statistical equation is produced.

We now develop the model to obtain a statistical equation.

The basic principle of the model is that, considering two forces that are experienced by $\mathrm{dN}$, the expansive internal force in the spherical shell and the compressive force immediately outside the shell are proportional at $\mathrm{d} N$.

The type of the expansion force differs among cases, but the compressive force is always assumed to be determined by the temperature $T_{i}$ and $\mathrm{d} N$. As the expansion force, we here assume a Coulomb interaction force. We then have

Coulomb interaction energy $=\frac{3}{2} k_{B} T_{i} \times d N . \quad$ (principle equation)

As mentioned, the left-hand side is an expansion force, and the right-hand side is a compression force. To calculate the above-mentioned principle equation, we determine the Coulomb interaction energy and $\mathrm{d} N$ as follows. First, $\mathrm{d} N$ is represented as

$$
\mathrm{d} N=g f \mathrm{~d} \vec{k}=g f\left(\frac{1}{d \tau}\right),
$$

where $\tau, g$, and $f$ denote the volume,state number, and partition function, respectively.

This equation implies that thermal equilibrium is locally confined at $\mathrm{d} N$. 
The electrostatic energy (i.e., the Coulomb interaction energy) $U_{\mathrm{E}}$ is calculated as

$$
U_{E}=\frac{1}{2} \varepsilon_{0}\left(\frac{e}{4 \pi \varepsilon_{0} a_{i}^{2}}\right)^{2} d \tau
$$

where $\varepsilon_{0}$ denotes the permittivity in a vacuum, and $a_{i}$ denotes the radius of the spherical shell.

At this time, a volume element of the integral is represented as

$$
\mathrm{d} \tau=\frac{1}{\mathrm{~d} \vec{k}}=\frac{4 \pi}{3} a_{i}^{3} .
$$

Substituting this volume element into Eqs. (15) and (16), the principle equation is calculated, based on $\mathrm{d} N$ and $U_{\mathrm{E}}$. The following equation is then obtained:

$$
a_{i}^{2}=\frac{9 \varepsilon_{0}}{e^{2}}\left(3 k_{B} T_{i}\right) f g .
$$

This derived equation will be employed later in this paper.

\section{Theory}

\subsection{Mass of a magnetic flux quantum}

First, let us consider the mass of a magnetic flux quantum, which is essential to all following calculations. The mass (i.e., rest energy $V$ ) of a flux quantum is given by

$$
V=\frac{1}{2} \Phi_{0} I,
$$

where $\Phi_{0}$ and $I$ denote the quantized flux and surrounding current of a magnetic flux quantum, respectively.

The current is a function of the cyclotron angular frequency $\omega_{\mathrm{c}}$ :

$$
I=\frac{2 e}{T}=2 e \frac{\omega_{c}}{2 \pi}=e \frac{\omega_{c}}{\pi},
$$

where $e$ and $T$ denote the charge of an electron and the cyclotron period, respectively.

Moreover, we have

$$
\omega_{c}=B_{0} \frac{2 e}{m},
$$

and

$$
\Phi_{0}=\pi a^{2} B_{0} .
$$

Thus,

$$
\omega_{c}=\frac{\Phi_{0}}{\pi a^{2}} \frac{2 e}{m} .
$$

Substituting the above equation into the equation of the current $I$, we get

$$
V=\frac{1}{2} \Phi_{0}^{2} \frac{2 e^{2}}{\pi^{2} a^{2} m},
$$

where

$$
\Phi_{0}=\frac{h}{2 e} .
$$




\subsection{Application to the present case of many-body interaction of magnetic flux quanta}

\subsubsection{Critical temperature considering many-body interactions}

Our model is schematized in Fig. 6. This figure is analogous to Fig.5. However, note that the central magnetic flux quantum in the sphere shell has a magnitude. In this figure, parameter $a$ is the radius of a magnetic flux quantum, and $l_{i}$ is the lattice constant of the magnetic flux quanta. This paper claims that a magnetic flux quantum radius can vary, but the flux must remain at $h / 2 e$. Therefore, the larger the magnetic field, the greater the density of the magnetic flux quanta as $a$ decreases. At the transition $T=T_{\mathrm{c}}$, we have

1) $\mathrm{a} \equiv l_{i}$, meaning that the radius of a magnetic flux quantum equals the lattice constant of the magnetic flux quanta.

2) The lattices of the magnetic flux quanta are simultaneously melted when a magnetic flux quantum collapses.

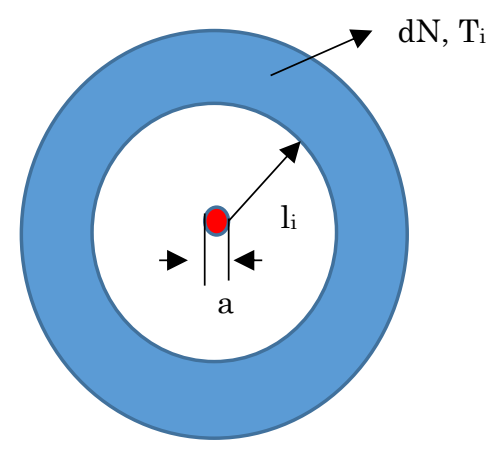

Fig. 6

Schematic of the model of many-body interactions in the case of magnetic flux quanta

First, we construct a spherical shell of radius $l_{i}$ containing a magnetic flux quantum of radius $a$ along with differential numbers $\mathrm{d} N$ of other magnetic flux quanta and a local temperature $T_{i}$. Note that, distinct from Fig. 5 , the central magnetic quantum has magnitude (i.e., the radius). The spherical shell, $\mathrm{d} N$, receives a compressive force from the outside and a simultaneous expansive force exerted by interactions with the central magnetic flux quantum. These two forces are propotional at $\mathrm{d} N$. Based on this proportionality principle, we can derive a statistical equation considering the many-body interactions. Moreover, $a$ and $l$ i are equal at the transition point. 
Adapting Fig. 5 in the review section to the present case (Fig. 6) and attributing the repulsive forces of each magnetic flux as Coulomb interaction between the Cooper pairs surrounding a magnetic flux quantum, we obtain the following equation:

$$
l_{i}^{2}=\frac{9 \varepsilon_{0}}{e^{2}} 3 k_{B} T_{i} f g,
$$

where the partition function is approximated by Boltzmann statistics:

$$
f=\frac{n}{n_{0}} \exp \left(-\frac{V}{k_{B} T_{i}}\right) .
$$

The transition point is determined by taking the extremum of Eq. (26):

$$
\frac{d l^{2}}{d T}=\frac{d}{d T}\left[\frac{9 \varepsilon_{0}}{e^{2}} 3 k_{B} T g f_{0} \exp \left(-\frac{V}{k_{B} T}\right)\right] \equiv 0,
$$

where

$$
\frac{n}{n_{0}} \equiv f_{0}
$$

After performing the calculation, we get

$$
1+T\left(\frac{V}{k_{B} T^{2}}\right)=0
$$

The sign (+,-) of the energy gap is minus, and the absolute value is

$$
T \equiv T_{C}=\frac{V}{k_{B}}
$$

That is

$$
T_{C}=\frac{1}{k_{B}}\left(\frac{1}{2} \frac{2 e^{2}}{\pi^{2} a^{2} m} \Phi_{0}^{2}\right)
$$

where

$$
\Phi_{0} \equiv \frac{h}{2 e}
$$

or

$$
a^{2}=\frac{2 e^{2} \Phi_{0}^{2}}{2 \pi^{2} m k_{B}} \frac{1}{T_{c}}
$$

The above Eq. (31) is a very important result that a superconducting energy gap is essentialy equal to the rest energy of a magnetic flux quantum as a result of considering the many-body interactions.

\subsubsection{Critical magnetic field at each critical temperature}

Let us now consider the critical magnetic field $B_{c}$. In our previous paper [10], the critical current density $j_{c}$ under a magnetic field was derived as 


$$
j_{c}=j_{c 0} \exp \left[-\left(\frac{e a^{2}}{\hbar} B_{r}+\frac{e a d}{\hbar} B_{S}\right)\right]
$$

The anisotropic properties are averaged by the following equations:

$$
B_{s}=B_{r} \equiv \frac{B}{2}
$$

and

$$
a \equiv d .
$$

Defining the critical magnetic field as the magnetic field $B$ at which the exponential function of the critical current density equation becomes $e^{-1}$, we have

$$
B_{c}=\frac{\hbar}{e a^{2}} \text {. }
$$

That is,

$$
B_{C}=\frac{\hbar}{e}\left(\frac{e^{2}}{2 \pi^{2} m k_{B}} 2 \Phi_{0}^{2} \frac{1}{T_{C}}\right)^{-1} .
$$

Importantly, this critical magnetic field implies thermal equilibrium; that is, no current flows. It is sufficient to consider the critical magnetic field at zero temperature.

\subsubsection{Inherent critical current density $j_{c 0}$ at each critical temperature}

In our previous paper [10], the wave function of an electron at the transition was given as

$$
\psi=|\Phi| \exp \left(-\frac{e B a}{2 \hbar}|x|\right) .
$$

Normalizing this wave function to

$$
\int \psi^{2} d x d S=1
$$

we can write

$$
S|\Phi|^{2} \int_{-\infty}^{\infty} \exp \left(-\frac{e B a}{2 \hbar}|x| \times 2\right) d x=1
$$

That is,

$$
2 S|\Phi|^{2} \frac{\hbar}{e B a}=1,
$$

where

$$
\mathrm{S} \equiv \frac{1}{k^{2}} .
$$

In this equation, $k$ is the wavenumber derived in [10].

Thus we have

$$
S=\left(\frac{2 \hbar}{e B a}\right)^{2} .
$$

Accordingly

$$
|\Phi|^{2}=\frac{1}{2}\left(\frac{e B a}{\hbar}\right)^{3} .
$$


The inherent critical current density is derived as

$$
j_{c 0}=e|\Phi|^{2} v=\frac{1}{2} e v\left(\frac{e B a}{\hbar}\right)^{3},
$$

where the velocity $v$ is obtained as

$$
v=\frac{\hbar k}{m}=\frac{\hbar}{m}\left(\frac{e B a}{2 \hbar}\right) .
$$

We then have

$$
j_{c 0}=\frac{e \hbar}{2 m} \times 2^{3} \times\left(\frac{e B}{2 \hbar}\right)^{4} a^{4} .
$$

Considering Maxwell's equation

$$
\frac{\partial B}{\partial R}=\mu_{0} j_{c 0}
$$

we can write

$$
B=\mu_{0} j_{c 0} R
$$

assuming that

$$
R \equiv a
$$

Thus we have

$$
j_{c 0}=2^{3} \frac{e \hbar}{2 m}\left(\mu_{0} j_{c 0} a\right)^{4}\left(\frac{e}{2 \hbar}\right)^{4},
$$

whereby

$$
j_{c 0}^{3}=\left(\frac{2 m}{2^{3} e \hbar}\right)\left(\frac{2 \hbar}{e}\right)^{4} \frac{1}{\mu_{0}} \frac{1}{a^{8}} .
$$

Fig. 7 illustrates the physical meaning of the inherent critical current density $j_{c 0}$.

Note that, as $j_{c 0}$ in Eq. (52) does not depend on the magnetic field $B$, it must distribute at the center point of the cross section of a superconducting wire (Fig.7).

Fig. 7

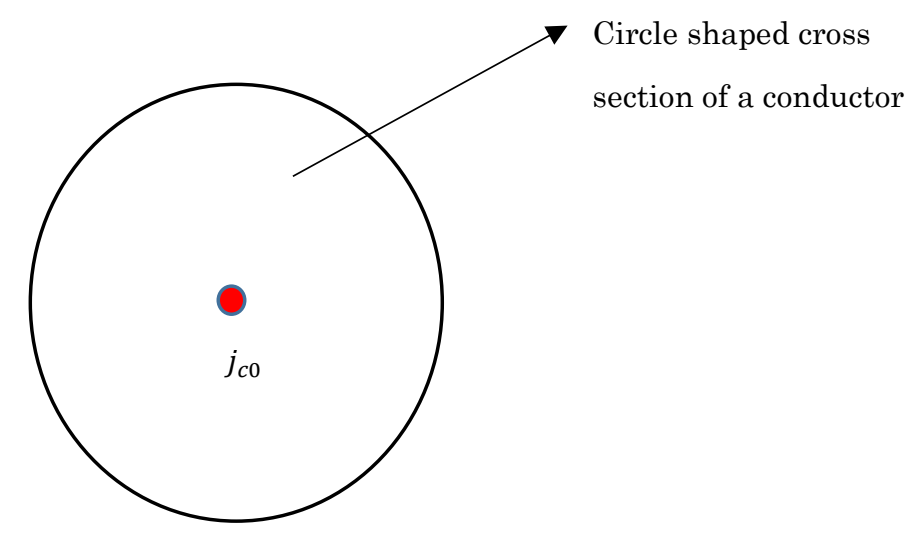

Physical interpretation of the inherent critical current density: $j_{c 0}$

locates at the central point of the cross section of a conductor. Therefore, it receives no influence from magnetic fields. 


\subsubsection{Pinning critical current density with a self-magnetic field}

In [10], we derived the following expression:

$$
j_{c}=j_{c 0} \exp \left[-\left(\frac{e a^{2}}{\hbar} B_{r 0}+\frac{e a d}{\hbar} B_{z 0}\right)\right],
$$

where $B_{r 0}$ and $B_{z 0}$ are the self-magnetic field components in the $r$ and $z$ directions, respectively.

In this paper, the anisotropic properties are averaged as

$$
B_{r 0}=B_{z 0}=\frac{1}{2} B_{0}
$$

and

$$
a=d
$$

Equation (55) then becomes

$$
j_{c}=j_{c 0} \exp \left[-\frac{e a^{2}}{\hbar} B_{0}\right]
$$

Applying Maxwell's equation

$$
\frac{\partial B_{0}}{\partial R}=\mu_{0} j^{\prime}
$$

we get

$$
j_{c}=j_{c 0} \exp \left[-\frac{e a^{2}}{\hbar} \mu_{0} j^{\prime} R\right] .
$$

To obtain the critical magnetic field $B_{c, p}$ of pinning, we set the exponential function to $e^{-1}[10]$ and replace the radius $a$ in Eq. (58) with the pinning center $a_{p}$ :

$$
B_{c, p}=\frac{\hbar}{e a_{p}^{2}} \text {. }
$$

Similarly, setting the exponential function as $e^{-1}$ and replacing the radius $a$ with that of a pinning center in Eq. (60), we obtain the pinning critical current density $j_{c, p}$ corresponding to the critical magnetic field $B_{c, p}$. We then have

$$
\frac{e a_{p}^{2}}{\hbar} \mu_{0} j_{c, p} R \equiv 1
$$

That is,

$$
j_{c, p}=\frac{\hbar}{e a_{p}^{2}} \frac{1}{\mu_{0}} \frac{1}{R}
$$

Although pinning centers and the captured magnetic flux quantum have no net kinetic energy, in our model of a sphere shell, internal expansion force, which comes from the Coulomb interaction of Cooper pairs surrounding a magnetic flux quantum, still proportional to outer thermal compressive force at $\mathrm{dN}$. We consider this outer temperature to be continuous for applied temperature T.Note that, here, it is assumed that the pinning centers are distributed uniformly and that the lattice constant $l_{\mathrm{i}}$ 
equals to the radisu $a_{p}$ of a pinning center. This lattice constant $l$ i still varies, depending on a magnetic field becuser the larger the magnetic field, the greater the density of magnetic flux quanta is. Therefore, considering that the lattice constant becomes equal to the radius of pinning center and similarly to Eq. (26), we obtain the following equation for pinning:

$$
a_{p}^{2}=\frac{9 \varepsilon_{0}}{e^{2}} 3 k_{B} T f g
$$

where

$$
f=f_{r 0} \exp \left(-\frac{U_{P}}{k_{B} T}\right)
$$

In this equation, $U_{P}$ is the potential of the pinning center. Contrary to magnetic field transitions and inherent critical currents, the magnetic flux quanta have no momenta because the pinning centers tie them strongly. Therefore, the number $\mathrm{d} N$ is independent of temperature, implying that the partition function Eq. (65) is constant. This implies that this case claims that there are no interactions between magnetic flux quanta. We then set $T=T_{c}$ in Eq. (65) as follows:

$$
f=f_{r 0} \exp \left(-\frac{U_{P}}{k_{B} T_{c}}\right) .
$$

The pinning critical current becomes

$j_{c, p}=\frac{\hbar}{e} \frac{1}{\mu_{0}} \frac{1}{\lambda_{L}} \frac{e^{2}}{9 \varepsilon_{0}} \frac{1}{3 k_{B}} \frac{1}{g f_{r 0}} \frac{1}{T} \exp \left(\frac{U_{p}}{k_{B} T_{C}}\right)$.

In this equation, $g f_{r 0}$ defines the density of magnetic flux quanta at the transition point. Because the magnetic flux quanta is destroyed at this critical point [10], $g f_{r 0}$ was assumed as unity (i.e., the number 1), and the London length $\lambda_{\mathrm{L}}$ substituted $\mathrm{R}$.

\section{Results}

First of all, all calcaltions conducted in this paper employed the MS excel software.

As shown in Fig. 8, the critical magnetic field was a linear function of critical temperature. The critical magnetic field differs among superconductors with different inherent critical temperatures. After reviewing the literature on critical magnetic fields in various superconductors [14-17], we concurred that the theory sufficiently agreed with the experimental results.

Figure 9 plots the inherent critical current density as a function of critical temperature. The relationship is monotonically increasing but not linear.

Figure 10 plots the pinning critical current density as a function of applied temperature $T$. This pinning critical current density cannot be defined under a uniform magnetic field, i.e., the self-field is not a uniform magnetic field; it simply depends on the applied temperature. In this example, the critical temperature $T_{c}$ was assumed as $90 \mathrm{~K}$ and the London length $\lambda_{\mathrm{L}}$ was assumed as $2.0 \times 10^{-6} \mathrm{~m}$, characterizing highTc cuprates. Moreover, pinning potential here is assumed to be $5.5 \times 10^{-20} \mathrm{~J}$. As a result of our 
literature reviews [18], experiments of high-Tc cuprates regarding pinning critical current densities with self-fields are well suited for the present theory.

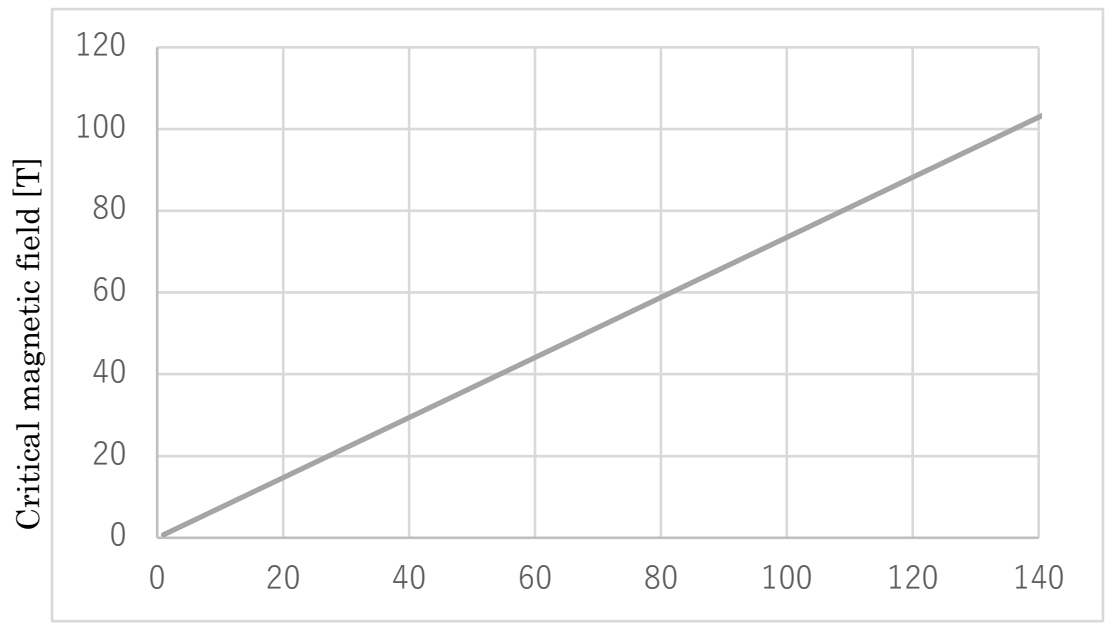

Critical temperature $[\mathrm{K}]$

Fig. 8

Critical magnetic field vs. critical temperature.

Note that the horizontal axis is the critical (not the applied) temperature, and at the critical magnetic field, the state transitions from normal to superconducting

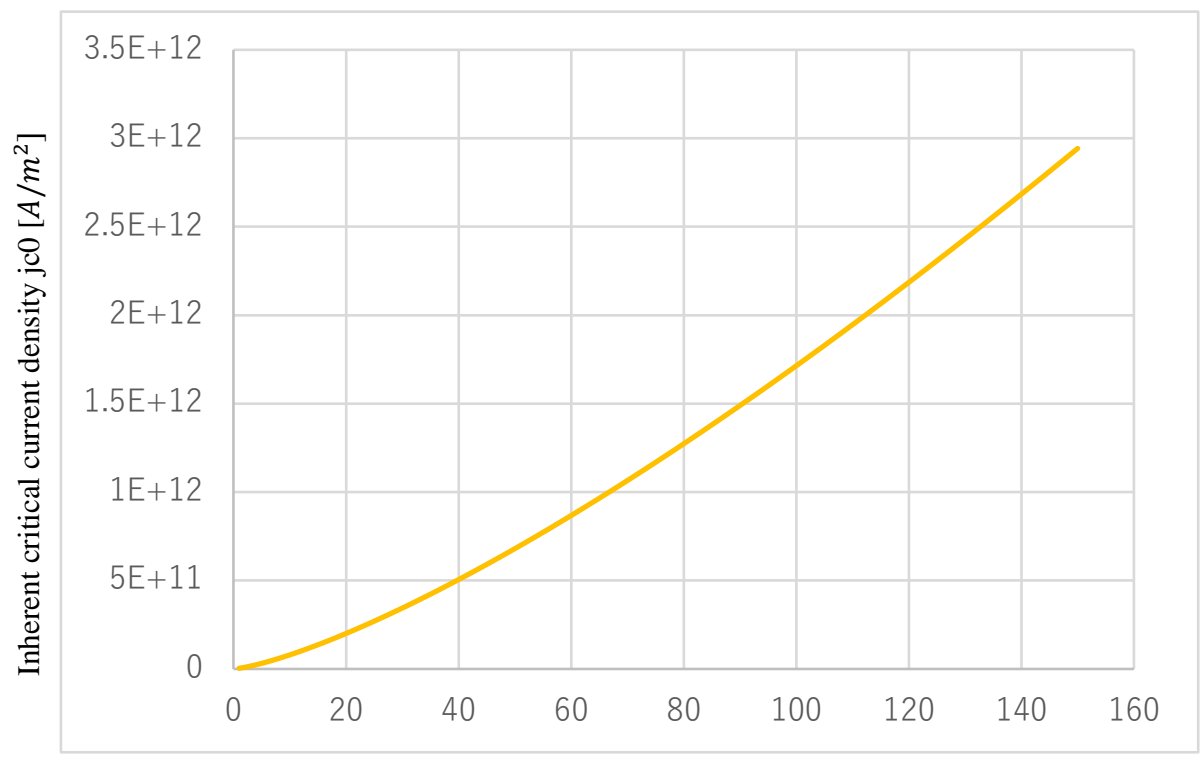

Critical temperature $[\mathrm{K}]$

Fig. 9

Inherent critical current density vs. critical temperature. 
The inherent critical current density flows along the central line of a conductor, so the magnetic field is irrelevant (note that this situation differs from the pinning critical current density with self-magnetic fields)

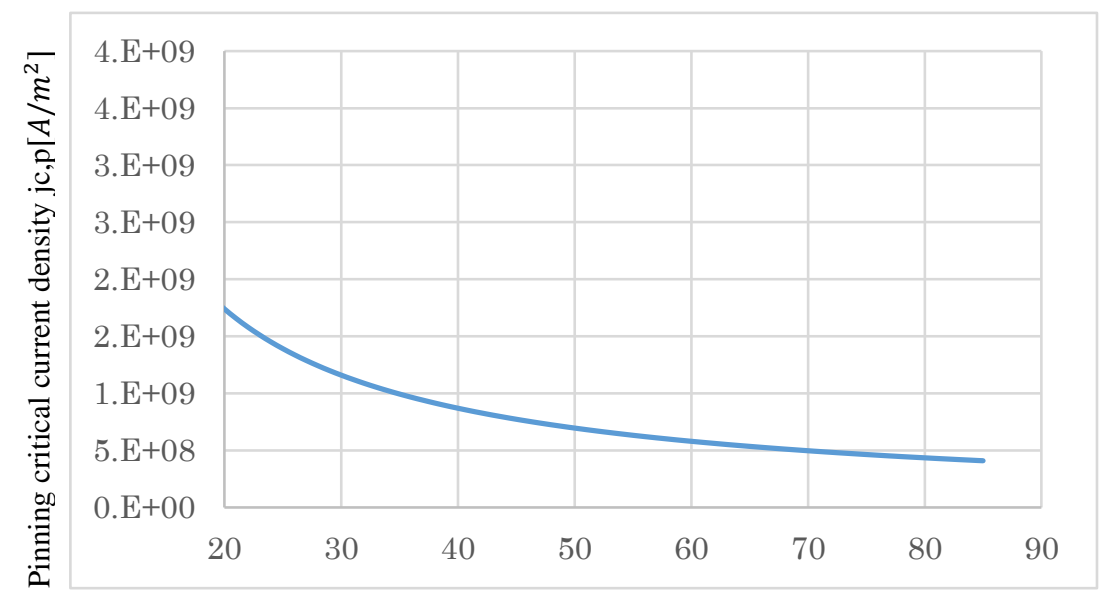

Temperature $\mathrm{T}[\mathrm{K}]$

Fig. 10

Pinning critical current density vs. applied temperature $T$.

The horizontal axis is the applied temperature (not the critical temperature), and the pinning critical current density has self-magnetic fields.

\section{Discussion}

\subsection{Unresolved problems in the conventional pinning theories}

The conventional pinning theories are based wholly on classical mechanics. These theories consider three types of interactions:

1) Core interactions,

2) Magnetic field interactions,

3) Thermal excitation and interactions.

In the core interactions, the pinning center collectively captures the magnetic flux quanta. The magnetic field interactions (implying interactions between the magnetic flux quanta) are collectively described by elastic constants. The thermal interactions (which also imply multiple interactions between the magnetic flux quanta) are tied to the magnetic field interactions. However, conventional pinning theories are problematic for several reasons. First, they cannot describe the dynamics of magnetic flux quanta, which require full consideration of many-body interactions. To acquire the pinning forces, the critical current density in the conventional approach is derived from critical current 
measurements rather than pure theory. Moreover, the models claim to provide the resistivity but the resistivities do not reflect the real superconductivity because they neglect the critical temperature. On the other hand, our model, which includes the many-body interactions, theoretically provides the critical current without requiring numerical or fitting calculations. Our critical currents depend on both the critical magnetic field and critical temperature.

\subsection{Our physical picture of magnetic field transitions}

Our present calculations revealed the physical meaning of magnetic field transitions. Because the radius of a magnetic flux quantum can vary but the quantized flux must remain at $h / 2 e$, density of magnetic flux quanta also varies. Our model reflected this fact. Our many-body interaction model predicted that a magnetic flux quantum simultaneously collapses during, melting of the lattice of magnetic flux quanta.

\subsection{The versatility of our method for many-body interactions}

In this paper, the many-body problem for magnetic flux quanta was demonstrated in a spherical shell model. However, this model is quite versatile and (as demonstrated in our previous paper) can reproduce the behaviors of high-Tc cuprates and accurately predict Curie temperatures [5].

\subsection{Significances of this paper}

Let us consider the significances of this paper:

\section{1) The critical current is theorized without fitting methods or numerical calculations}

As mentioned above, the pinning forces in a superconducting material have been measured by measuring the critical current. However, this approach is insufficient because the purpose of acquiring the pinning-force properties is to obtain the critical current. Thus far, research on magnetic flux quantum dynamics has not solved the many-body interactions of magnetic flux quanta, which are essential for calculating the critical current density. Our paper, in turn, succeeded theoretically in deriving critical current density with full consideration of many-body interaction of magnetic flux quanta.

\section{2) The superconducting transition under magnetic fields was clarified}

In the conventional view, magnetic flux quanta move under the Lorentz force generated in a sea of currents. Then the movement of a magnetic flux quantum generates a voltage by electromagnetic induction. However, when the Lorentz forces are applied to the critical current phenomenon, this approach violates the third law of Newtonian mechanics. When magnetic flux quanta move under 
Lorentz forces, which points in the current sea respond with a reaction force cannot be discerned. Instead of the Lorentz force movments, we now suggest diffusion movements that originate from the interactions between magnetic flux quanta [10]. Indeed, a magnetic-field-induced transition results from many-body interactions and the destruction of magnetic flux quanta. This occurs simultaneously with the melting of the lattice of magnetic flux quanta. This fact can only be explained by elementary quantum mechanics, not by classical mechanics. Our present interpretation is consistent with experimental results.

\section{3) The relationships among critical current, critical magnetic field and critical temperature were revealed theoretically}

The relationships among critical current, critical magnetic field, and critical temperature have been well documented in experiments and have been replicated by fitting or numerical methods [9]. However, data fitting and numerical methods cannot clarify the physical meaning of these relationships. We clarified that the transition under a magnetic field involves destruction of the magnetic flux quanta accompanied by melting of their lattice through our purely theoretical analysis. This theoretical approach must consider the many-body interactions among the magnetic flux quanta.

\section{Conclusion}

Adapting our original generalized method to many-body interactions, we solved the many-body interactions of magnetic flux quanta. First, we calculated the critical magnetic field at each critical temperature. After obtaining the inherent critical current density at the critical temperature, we then derived the pinning-related critical current density with self-magnetic fields. The obtained results agreed with experimental observations, implying that our method resolves the problems associated with magnetic flux quanta.

As mentioned, the pinning critical current density depends on the applied temperature and on the magnitude and angle of the applied magnetic field. We will derive an equation for the pinning critical current density that incorporates these three variables in future work. In the literature [9], this goal was achieved in part by fitting and numerical methods. Instead, we shall examine whether the radius $a$ of the magnetic flux quantum should be replaced by that of the pinning center $a_{p}$ in Eq. (13). Although experiments by other reaserchers could measure all dependences of the critical current in HTS wire, the data must be systematically organized by us, which is time-consuming. Thus, in the future, we will derive an equation that simultaneously relates the critical current density to applied temperature, magnetic field magnitude, and magnetic field angle and test its agreement with experimental results. 


\section{Additional information}

This paper is not related to any competing interests such as funding, employment, and personal financial interests. Moreover, this paper is not related to non-financial competing interests.

\section{Acknowledgement}

We thank Enago (www.enago.jp) for English language Review.

\section{References}

[1] J. Bardeen, L.N. Cooper and J. R. Schrieffer, Phys. Rev. 108 (5), 1175-1204 (1957)

[2] J.G. Bednorz and K.A. Müller, Z. Physik B-Condensed Matter. 64, 189-193 (1986)

[3] J. Nagamatsu, et al, Nature, 410, 63 (2001)

[4] Y. Kamihara, et al, J. Am. Chem. Soc, 128 (31), 10012-10013 (2006)

[5] S. Ishiguri, "Analytical Descriptions of High Tc Cuprates by Introducing Rotating Holes and a New Model to Handle Many-Body interactions," Preprints 2020, 2020050105 (doi: 10.20944/preprints202005.0105.v2)

[6] M. Somayazulu, et al, Phys. Rev. Lett. 122, 027001 (2019)

[7] J. Pitel and P. Kovac, Supercond. Sci. Technol. 10, 847 (1997)

[8] M. Yamamura, et al, Technology of Superconductivity, Institute of Electrical Engineers of Japan, Tokyo, pp. 24-26 (2001)

[9] T. Kiss, $7^{\text {th }}$ seminar for young researchers in superconductor and cryogenics fields by the branch of Tohoku and Hokkaido in Cryogenics and Superconducting Societies of Japan, 75-107 (2002)

[10] S. Ishiguri and T. Sato, Phys. C 469, 135-138 (2009)

[11] H. Mukuda, et al, Phys. Rev. Lett. 96, 087001 (2006)

[12] Y. Kohsaka, et al, Science, 315, 1380 (2007)

[13] J. Ri et al, J. Cryog. Supercond. Soc. Jpn. 44 (12), 562 (2009)

[14] A.Y. Ganin, et al, Nat. Mat. 7, 367 (2008)

[15] Y. Kasahara et al, 69th Phys. Soc. Jpn. 4,822 (2014)

[16] M. Zehetmayer, et al, Phys. Rev. B66, 052505 (2002)

[17] V. Braccini, et al, Phys. Rev. B71, 012504 (2005)

[18] K. Hayashi, Jpn. App. Phys. 79 ( 1), 3-13 (2010) 